Copyright $(2005$ IEEE. Reprinted from IEEE Signal Processing Letters, Vol. 12, Issue 2, pp. 126-129, February 2005.

This material is posted here with permission of the IEEE. Such permission of the IEEE does not in any way imply IEEE endorsement of your university's web sites products or services. Internal or personal use of this material is permitted. However, permission to reprint/republish this material for advertising or promotional purposes or for creating new collective works for resale or redistribution must be obtained from the IEEE by writing to pubs-permissions@ieee.org.

By choosing to view this document, you agree to all provisions of the copyright laws protecting it. 


\title{
Spatial Filter-Based Absorbing Boundary for the 2-D Digital Waveguide Mesh
}

\author{
Antti Kelloniemi, Lauri Savioja, Member, IEEE, and Vesa Välimäki, Senior Member, IEEE
}

\begin{abstract}
The digital waveguide mesh is a method for simulating wave propagation, for example, in an acoustic system. Research on the boundary conditions has been going on for years, but adequate solutions for absorbing boundaries have not yet been presented for the digital waveguide mesh. In this work, a new method for constructing absorbing boundaries for a two-dimensional (2-D) rectangular mesh is introduced. With the use of the proposed numerically optimized spatial filtering with an interpolated mesh structure, the reflection was diminished to under $-25 \mathrm{~dB}$ at incidence angles $|\theta| \leq 79.26^{\circ}$ on a frequency band limited only at the very lowest and highest ends.
\end{abstract}

Index Terms-Acoustic propagation, acoustic signal processing, boundary conditions, digital waveguide mesh, FDTD methods.

\section{INTRODUCTION}

$\mathbf{T}$ HE digital waveguide mesh is a finite difference time-domain modeling technique, which provides a numerical solution to the wave equation in multiple dimensions, and thus, it has the benefit of including diffraction and interference effects into the model [1]-[3]. The method has been extended to two and three dimensions from the digital waveguide method used for one-dimensional (1-D) simulation of musical instruments, such as plucked string instruments and woodwinds [4].

The original rectangular mesh suffers from direction-dependent dispersion [1], [2]. This can be reduced by using different mesh topologies or interpolation and frequency warping techniques [5]. These methods enhance the behavior of the mesh as a model of wave propagation in a homogenous medium. The dispersion error also affects the reflections at the boundaries.

Approximation methods of reflecting boundary conditions have been evaluated in a previous study [6]. For practical simulation of acoustic spaces, a controllable boundary model is needed, which allows arbitrary frequency-dependent reflection. Absorbing boundaries are needed for mesh truncation, when calculations of infinite space are made using finite mesh sizes. In this case, maximal absorption is needed at all angles of incidence.

The original absorbing boundary condition is known to give rise to unwanted reflections [3]. Subsequent improvements were

\footnotetext{
Manuscript received April 20, 2004; revised August 5, 2004. This work was supported by the Academy of Finland under Project 201050. The associate editor coordinating the review of this manuscript and approving it for publication was Dr. Steven L. Grant.

A. Kelloniemi and L. Savioja are with the Telecommunications Software and Multimedia Laboratory, Helsinki University of Technology, FIN-02015 HUT, Finland (e-mail: antti.kelloniemi@hut.fi; lauri.savioja@hut.fi).

V. Välimäki is with the Laboratory of Acoustics and Audio Signal Processing, Helsinki University of Technology, FIN-02015 HUT, Finland (e-mail: vesa.valimaki@hut.fi).

Digital Object Identifier 10.1109/LSP.2004.840917
}

based on a Taylor-series expansion [7], but they, too, had the drawback of a limited incidence angle. The new method introduced in this letter is based on expanding the area of calculation using spatial filtering at the boundary. The weighting coefficients are optimized numerically.

In Section II of this letter, the topology of the rectangular digital waveguide mesh is briefly described. The theory of absorbing boundaries is discussed in Section III. Simulation results are presented in Section IV using the interpolated rectangular mesh in two dimensions. Section V concludes the paper.

\section{InTERPOLATED Multidimensional WAVEGUide MeSh}

A multidimensional rectangular digital waveguide mesh is a regular array of nodes arranged along each perpendicular dimension, interconnected by 1 -D digital waveguide segments. A difference equation for the nodes of an $\mathrm{N}$-dimensional rectangular mesh is [3]

$$
p_{c}(n)=\frac{1}{N} \sum_{l=1}^{2 N} p_{l}(n-1)-p_{c}(n-2)
$$

where $p$ represents the pressure at a junction at time step $n$, subscript $c$ denotes the junction to be calculated, and index $l$ denotes its $2 N$ axial neighbors.

In the interpolated mesh, there are also connections from a node to all its diagonal neighbors. As a result, the direction dependence of dispersion error created in the rectangular mesh is effectively diminished. A difference equation for the nodes of a two-dimensional (2-D) interpolated rectangular mesh is

$$
p_{c}(n)=\frac{1}{4} \sum_{l=1}^{3} \sum_{k=1}^{3} h_{l, k} p_{l, k}(n-1)-p_{c}(n-2)
$$

where $h_{l, k}$ are the weighting coefficients for each node [5].

The dispersion behavior of the model could be improved further by frequency warping [5]. The warping technique was not used in the simulations and optimizations run for this letter, but the optimized solution was also tested with the frequencywarped mesh.

\section{AbSORbing Boundary CONDITIONS In the Digital WAVEGUIDE MESH}

\section{A. Previous Methods}

The basic absorbing boundary condition is constructed by adding one extra node outside the mesh. The equation for up- 
dating the node value is derived from the discrete boundary condition of the acoustical pressure

$$
p_{B}(n)=(1+r) p_{1}(n-1)-r p_{B}(n-2)
$$

where subscript $B$ denotes the additional boundary node, and subscript 1 represents its perpendicular neighbor on the edge of the mesh [3]. The two terms on the right-hand side in (3) represent the sound pressure in front of the boundary one time step ago and the sound pressure reflected from the boundary two time steps ago, respectively. Hence, with reflection coefficient value $r=0$, the last term is absent, and we get the basic absorbing condition

$$
p_{B, \mathrm{abs}}(n)=p_{1}(n-1) .
$$

It has been noticed in simulations that better absorption is needed for creating an adequate nonreflecting boundary [6]. One proposed solution is to approximate the wave equation in the form of calculating the boundary node value $p_{B, a b s}(n)$ as a Taylor series of the past value of the node $p_{1}(n-1)$ next to the boundary [7]

$$
\begin{aligned}
& p_{B, \mathrm{abs}}(n)=p_{1}(n-1)+\Delta n p_{1}^{\prime}(n-1)+\frac{\Delta n^{2}}{2 !} p_{1}^{\prime \prime}(n-1) \\
&+\frac{\Delta n^{3}}{3 !} p_{1}^{\prime \prime \prime}(n-1)+\cdots
\end{aligned}
$$

where $\Delta n$ is the temporal step size. The basic boundary condition (4) is equal to the zero-order solution of the series (5). In a previous study [6], the second-order Taylor-series approximation

$$
p_{B, \text { abs }}(n)=\frac{5}{2} p_{1}(n-1)-2 p_{2}(n-2)+\frac{1}{2} p_{3}(n-3)
$$

proposed by Murphy and Mullen [7] was chosen as the reference. Two additions and three multiplications are required for each boundary node per sample.

The main problem of both one-way approximation methods is their high dependency on the incident angle of the pressure wave. As the solution of the derivatives in (5) is 1-D, only the direction of incidence perpendicular to the boundary is considered.

\section{B. New Method}

As a new solution, we introduce a spatial averaging filter in the calculation of $p_{B}$. In addition to the values of the nodes on a line perpendicular to the boundary, their nearest parallel neighbors are also considered. Good results have been obtained by using three perpendicular nodes and the parallel neighbors of the two nearest to the boundary

$$
\begin{aligned}
p_{B, \mathrm{abs}}(n)= & h_{B a 1} p_{1, x}(n-1)+\frac{h_{B d 1}}{2} \\
& \times\left[p_{1, x-1}(n-1)+p_{1, x+1}(n-1)\right] \\
& +h_{B a 2} p_{2, x}(n-2) \\
& +\frac{h_{B d 2}}{2}\left[p_{2, x-1}(n-2)+p_{2, x+1}(n-2)\right] \\
& +h_{B a 3} p_{3, x}(n-3)
\end{aligned}
$$

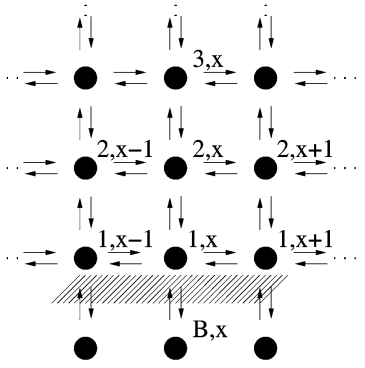

(a)

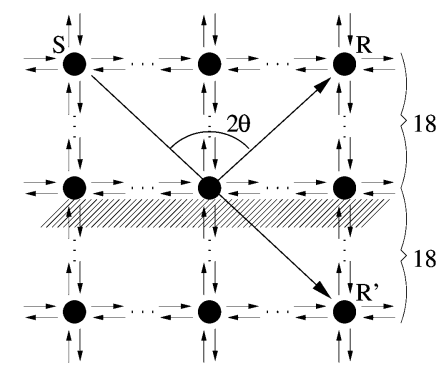

(b)
Fig. 1. Spatial filter nodes and the test setup shown for simplicity in the noninterpolated 2-D mesh. (a) Nodes involved in (7). (b) Source (S) and the receiver $(\mathrm{R})$ were placed in line, and the reflection from the boundary was compared to the level of the freefield signal measured at point $R^{\prime}$. The shaded area represents the location of the absorbing boundary.

where the subscripts of $p$ stand for the node coordinates, as indicated in Fig. 1 , and $h_{B a 1}, h_{B a 2}, h_{B a 3}, h_{B d 1}$, and $h_{B d 2}$ are the axial and diagonal filter coefficients. For the calculation, six additions and five multiplications are needed for each boundary node per sample. The use of the parallel neighbors of $p_{3, x}$ and the parallel node pairs one step further from $p_{2, x}$ and $p_{1, x}$ were tested in many combinations, but the results were not considerably improved. The values for the coefficients were optimized numerically for minimum reflection, as explained in Section IV.

\section{Simulation Setup and Results}

Simulations were executed in a 2-D rectangular interpolated mesh of $600 \times 300$ junctions. The mesh was initialized with a unit impulse filtered with the transfer function $1-z^{-2}$, as suggested by Karjalainen and Erkut [8], at the point $(280,18)$, and the simulation was run for 390 time steps. The receiver was located at the same distance from the boundary as the source, as indicated in Fig. 1(b). This way, the distance between the source and receiver was varied between 0 and 320 spatial sampling intervals, corresponding to the incidence angle $\theta \in\left[0,83.58^{\circ}\right]$. The level of the signal radiated directly from the source to the receiver was subtracted from the reflected signal. To resolve the absorption of the boundary, the resulting signal level of the reflection was compared to the level of a freefield signal that had passed the same distance in an unbounded mesh as the reflected signal had travelled from the source to the receiver, as illustrated in Fig. 1(b). The right half of the Hanning window function was used for windowing the last half of the received signal to avoid the truncation error in calculation of the spectra.

In Figs. 2-4, presenting the simulation results, a contour line is drawn at $A=-25 \mathrm{~dB}$, which corresponds to the absorbance of the walls in a regular anechoic room and was, therefore, chosen as the goal of adequate absorption. When using the basic absorbing condition (4), the simulation resulted in the reflection magnitude shown in Fig. 2. It is seen that sufficient attenuation is obtained only for some frequencies for values of the angle of incidence $\theta=\left[38.2^{\circ}, 53.8^{\circ}\right]$. Simulating the performance of the second-order Taylor-series solution gives the result shown in Fig. 3. It is seen that the area where the reflection magnitude is less than $-25 \mathrm{~dB}$ has grown remarkably, but still, the reflection is too large at incidence angles $\theta>68^{\circ}$. 


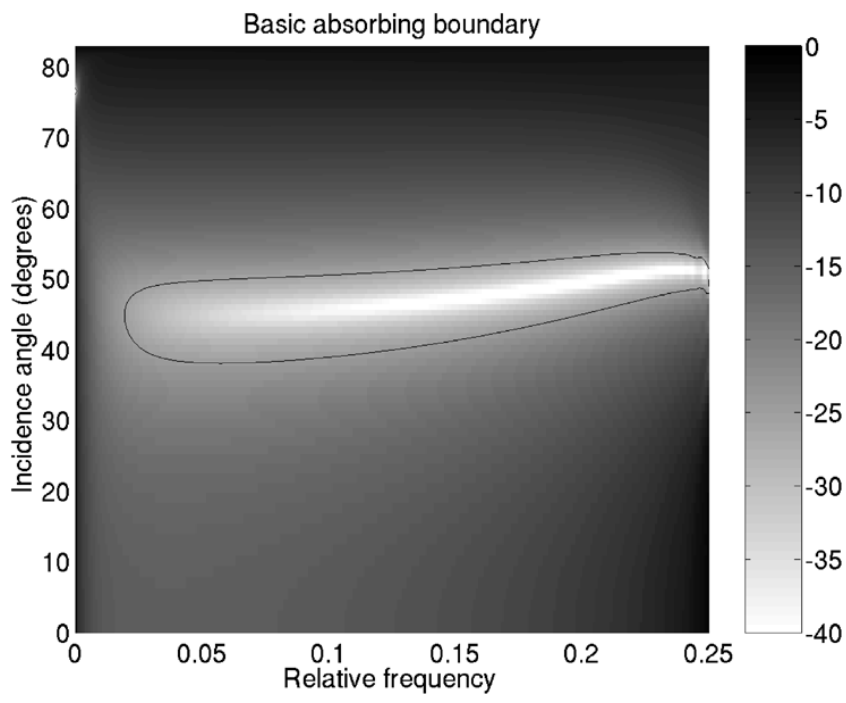

Fig. 2. Reflection magnitude of the basic absorbing boundary in decibels.

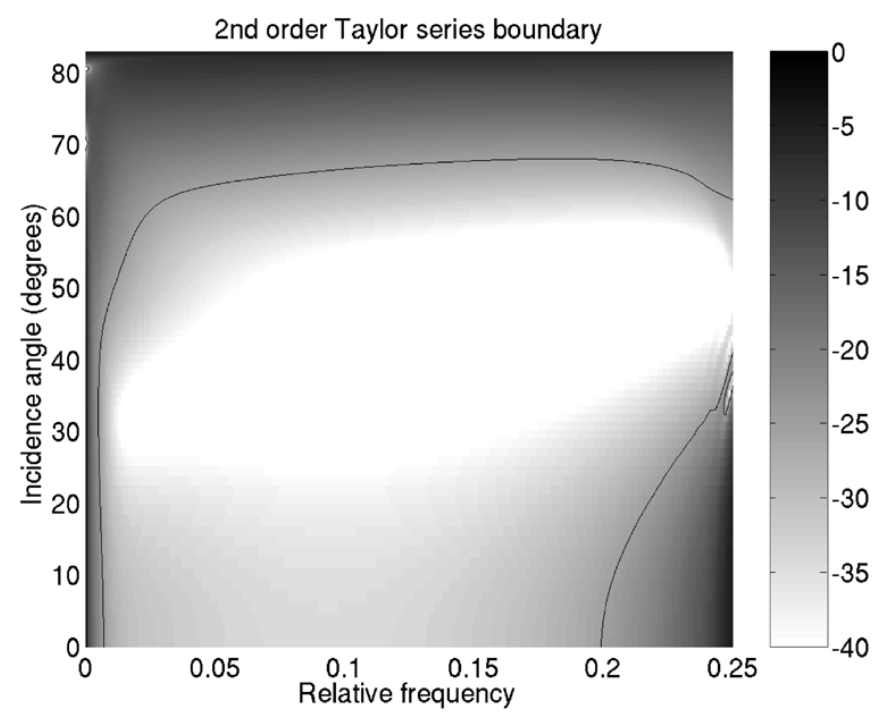

Fig. 3. Reflection magnitude of the second-order Taylor-series boundary in decibels.

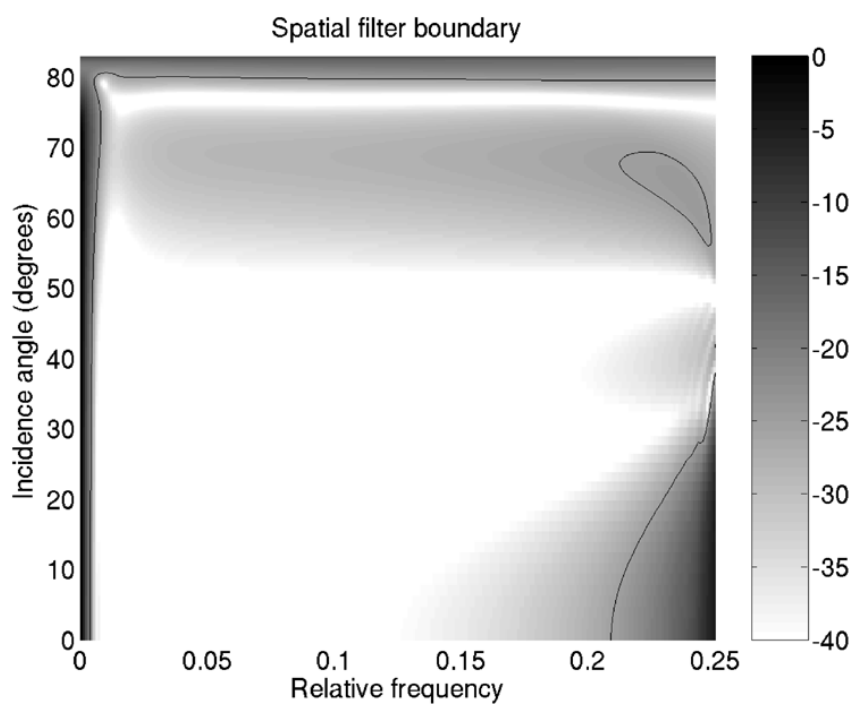

Fig. 4. Reflection magnitude of the numerically optimized spatial filter boundary in decibels.
As the optimization objective was to maximize the absorption, the sum of the reflected magnitude $A(\theta, f)$ in decibels was minimized. The sum was calculated over the area bounded by 211 equally spaced nodes within $\theta \in[0,80.3]^{\circ}$ and 213 equally spaced frequency points $f \in[0.01,0.22]$ relative to the sampling frequency. In general, the results obtained with the digital waveguide mesh are valid only up to the normalized frequency $f=0.25$. To avoid the influence of arbitrary points of high absorbance, all values below $A<-35 \mathrm{~dB}$ were counted in the sum as $A=-35 \mathrm{~dB}$. Optimization was done iteratively using the Nelder-Mead nonlinear algorithm in Matlab. For energy conservation, the sum of the coefficients was constrained at $[0,1]$. The initial values of the optimization were $h_{B a 1}=2.25$, $h_{B a 2}=-1.8, h_{B a 3}=0.5, h_{B d 1}=0.25$, and $h_{B d 2}=-0.2$. The values were chosen to be close to the Taylor-series constants, only giving $10 \%$ of the perpendicular node weights to their parallel neighbors. It was noted that the optimization results were highly dependent on the starting values. With these values, the optimization ended up with the best results out of the multiple tested choices.

The optimized coefficient values are $h_{B a 1}=2.42087845$, $h_{B a 2}=-2.33808068, h_{B a 3}=0.90809890, h_{B d 1}=$ 0.48591057 , and $h_{B d 2}=-0.47683624$. The resulting reflection magnitude is shown in Fig. 4.

The spatial filtering method can be used at relative frequencies $f=[0.0081,0.2088]$ in simulations where mesh truncation is needed, or there is other use for the absorbing boundary with $A<-25 \mathrm{~dB}$. At these frequencies, the largest incidence angle, where $A<-25 \mathrm{~dB}$, is $\theta=79.53^{\circ}$. With the use of frequency warping as post-processing for the resulting impulse response [5], the frequency band is extended to $f=[0.0081,0.2332]$.

The same method could be used with different mesh topologies as well. For example, it was tested for a 2-D rectangular noninterpolated mesh. With the same coefficient values, the largest usable incidence angle at the same frequencies was diminished to $\theta=67.48^{\circ}$, restricted by the reflection magnitude at high frequencies. It was noted that by restricting the simulation to relative frequencies $f<0.1$, the highest usable incidence angle could be moved further to the value $\theta=78.20^{\circ}$.

\section{CONCLUSION}

The high dependence on the incident angle has been one of the major drawbacks of the earlier methods of creating absorbing boundaries for the digital waveguide mesh. The optimized spatial averaging filter introduced in this letter has proven successful in solving this problem. The computational cost is about twice as large as in the previously proposed second-order Taylor-series solution, over which a significant improvement is obtained. The memory consumption of the two methods is equal.

\section{REFERENCES}

[1] S. Van Duyne and J. O. Smith, "The 2-D digital waveguide mesh," in Proc. IEEE Workshop Applicat. Signal Process. Audio Acoust., New Paltz, NY, Oct. 1993.

[2] — , "Physical modeling with the 2-D digital waveguide mesh," in Proc. Int. Comput. Music Conf., Tokyo, Japan, Sep. 1993, pp. 40-47. 
[3] L. Savioja, T. Rinne, and T. Takala, "Simulation of room acoustics with a 3-D finite difference mesh," in Proc. Int. Comput. Music Conf., Aarhus, Denmark, Sep. 1994, pp. 463-466.

[4] J. O. Smith, "Music Applications of Digital Waveguides," CCRMA, Stanford Univ., Stanford, CA, Tech. Rep. STAN-M-39, 1987.

[5] L. Savioja and V. Välimäki, "Reducing the dispersion error in the digital waveguide mesh using interpolation and frequency warping techniques," IEEE Trans. Speech Audio Process., vol. 8, pp. 184-194, Mar. 2000.

[6] A. Kelloniemi, D. T. Murphy, L. Savioja, and V. Välimäki, "Boundary conditions in a multi-dimensional digital waveguide mesh," in Proc. IEEE Int. Conf. Acoust. Speech Signal Process., vol. 4, Montreal, QC, Canada, May 2004, pp. 25-28.
[7] D. T. Murphy and J. Mullen, "Digital waveguide mesh modeling of room acoustics: Improved anechoic boundaries," in Proc. Int. Conf. Dig. Audio Effects, Hamburg, Germany, Sep. 2002, pp. 163-168.

[8] M. Karjalainen and C. Erkut, "Digital waveguides vs. finite difference structures: Equivalence and mixed modeling," EURASIP J. Appl. Signal Process., no. 7, pp. 978-989, 2004. 